\title{
Clinical Burden Related to Surgically Placed Feeding Enterostomy. A Single Center Study
}

\section{Muhammad Shadab Khan*, Syeda Sarah Batool, Shah Muhammad, Jahangir Farman Ali, Haider Mendhi and Abdaal Waseem Khan}

Department of GI and HBP Surgery, Sindh Institute of Urology and Transplantation, Karachi, Pakistan

*Corresponding Author: Muhammad Shadab Khan, Surgical Oncologist, Assistant Professor, Department of GI and HBP surgery, Sindh Institute of Urology and transplantation Karachi, Pakistan.
Received: July 10, 2021

Published: July 26, 2021

(C) All rights are reserved by Muhammad

Shadab Khan., et al.

\section{Abstract}

Objective: To determine the incidence and severity of postoperative complications following surgically placed feeding enterostomy. Introduction: Enteral nutrition is more advantageous compare to other forms of nutritional supplements. Enteral feeding tube placement is a relatively common general surgery procedure but there has been little standardization in their surgical technique and postoperative care. The aim of this study is to review indications, complications, and clinical burdens related to feeding tube placement and its long-term use.

Materials and Methods: Head and neck and upper gastrointestinal cancer patients presented in Sindh institute of urology and transplantation's Oncology center from November 2017 till October 2020 were reviewed retrospectively. Details reviewed from patient's files at the time of initial admission and subsequent follow ups during study period. All data was collected as per Proforma requirement in retrospective manner while maintaining strict confidentiality protocol according to institutional Ethical review board protocol. Collected data was analyzed for results using SPSS version 23.0.

Results: During this study period, a total of 116 patients underwent feeding enterostomies in our institute. Male patients were $51.7 \%$ and mean BMI was $17.86 \pm 2.43$. The mean age at the time of surgery was $44.22 \pm 14.02$ years.

Out of total 63 patients (54.3\%) develop complications. According to Clavein dindo classification 28.4\% minor while $25.8 \%$ major complications were noted.

Subgroup analysis showed that complications were more common in the feeding jejunostomy group with a statistically significant P-value.

Conclusion: Understanding the clinical burden and complications related with feeding tubes will help in minimizing associated morbidity and mortality. Feeding gastrostomy because of low clinical burden and complications should be preferred over feeding jejunostomy where possible.

Keywords: Feeding Enterostomy; Cancer; Complication; Clavein Dindo Classification

\section{Introduction}

Perioperative and long-term nutritional support directly impact survival and outcome in cancer patients. The critical role of adequate nutrition is well established in the cancer population.
The cachectic metabolic syndrome and loss of skeletal muscles in cancer patients predispose this population to a high risk of postoperative morbidities, delay recovery, and even mortality [1,2]. 
The risk of poor nutritional status and its consequences can be managed using enteral and parenteral nutrition. The benefits of enteral nutrition on healing, immune function, and nutritional built-up of cancer patients compared to other forms of nutritional supplements have been well established and most clinicians would agree that when feasible it is preferable over parenteral nutrition $[3,4]$.

Enteral nutrition can be established using natural orifices (nose/mouth) for a shorter duration or percutaneous for longterm access. For the long-term nutritional management of head and neck and upper gastrointestinal cancer patients feeding enterostomy (gastrostomy/jejunostomy) is the preferable route [5,6].

Although that enteral feeding tube placement is a relatively common general surgery procedure and most clinicians encounter challenging issues related to enteral tube management, there has been little standardization in their surgical technique and postoperative care [7-9].

\section{Aim of the Study}

This study's aim is to review indications, complications, and clinical burdens related to feeding tube placement and its longterm use.

\section{Materials and Methods}

A retrospective study was conducted in the Sindh Institute of urology and transplantation GI surgery department on patients with head and neck and upper GI cancers. All patients received in the Hanifa Suleiman Oncology center fulfilling inclusion criteria from November 2017 till October 2020 enrolled in the study. The Clinical record of all patients was retrieved from the Hanifa Suleiman Oncology clinic registry, GI clinic patient's registry, and hospital Record room. Variables mentioned in the questionnaire were all collected and stored with a given MR number maintaining data security and confidentiality. Approval for the study and exemption letter was obtained from Institutional Review Board (IRB) and the ethical review committee (ERC).

Details collected at initial admission of the patient in GI ward for surgery include patient age, gender, height, weight, Body mass index, total hospital stay which was recorded from the date of admission till the date of discharge, primary tumor, the procedure performed (feeding gastrostomy or feeding jejunostomy), treat- ment intent of primary cancer. Patient's first follow up in GI OPD recorded for the postoperative course and any further follow up visit in Outpatient or Emergency department for tube related complications were reviewed from files and severity of complication assessed according to Clavein dindo classification, need for readmission, and mortality associated with tube related complications was also reviewed. Subgroup analysis of the surgical procedures in terms of hospital stay, Outpatient and Emergency department visits, readmissions, mortality, and tube-related complications was also performed.

\section{Statistical analysis}

All data collected were analyzed using SPSS version 23.0. Frequencies and percentages were computed for categorical variables and postoperative complications while mean \pm standard deviation for quantitative variables. Chi-square or Fisher's Exact Test was used to determining the association between groups. An unadjusted odds ratio with $95 \%$ confidence interval was employed to assess the direction and magnitude of association between variables. Significance based on $\mathrm{p}$-value $<0.05$.

\section{Results}

During this study period, a total of 116 patients underwent feeding enterostomies in our institute. Among these surgeries 61 (52.5\%) were gastrostomies and 55 (47.4\%) were jejunostomies. Male patients were $60(51.7 \%)$ and mean BMI was $17.86 \pm 2.43$ (range $13.32-27.04 \mathrm{~kg} / \mathrm{m}^{2}$ ). The mean age at the time of surgery was $44.22 \pm 14.02$ years (range 12 - 85 years). The mean hospital stay for this surgical intervention was $4.27 \pm 3.2$ days (range 2 17 days). All patients included in the study were presented with the diagnosis of cancer in which 52 patients (44.8\%) had head and neck cancer while 51 patients (44.0\%) had esophageal and 13 patients $(11.2 \%)$ had gastric cancer. Indication for feeding tube placement was according to multidisciplinary team meeting recommendations where 24 tubes (20.7\%) were placed with palliative intent and 92 tubes (79.3\%) were placed with curative intent (surgery/ definite chemoradiotherapy).

Clinical burden and complications related to feeding tubes are summarized in table 1 . Out of 116 feeding enterostomies, 63 patients (54.3\%) develop complications during the follow-up period. According to Clavein dindo classification, 33 patients $(28.4 \%)$ had minor while 30 patients $(25.8 \%)$ had major complications. The most frequent complication encountered was wound infection 
in 34 patients (29.3\%) followed by GI symptoms in 25 patients (21.6\%) which include nausea, vomiting, diarrhea, and/or abdominal bloating. Feeding tubes related mortality was observed in 3 patients $(2.6 \%)$.

\begin{tabular}{|c|c|c|c|}
\hline \multicolumn{2}{|l|}{ Clinical burden } & \multirow{2}{*}{$\begin{array}{c}\mathbf{N}=116 \\
63\end{array}$} & \multirow{2}{*}{$\begin{array}{r}\text { (\%) } \\
54.3 \% \\
\end{array}$} \\
\hline Morbidity & Yes & & \\
\hline Mortality & Yes & 3 & $2.6 \%$ \\
\hline ER visit & Yes & 19 & $16.4 \%$ \\
\hline OPD visit & Yes & 25 & $21.6 \%$ \\
\hline Readmission & Yes & 12 & $10.3 \%$ \\
\hline Reoperation & Yes & 3 & $2.6 \%$ \\
\hline \multirow{3}{*}{$\begin{array}{l}\text { Clevien dindo classifica- } \\
\text { tion based morbidity }\end{array}$} & None & 48 & $41.4 \%$ \\
\hline & Minor & 33 & $29.3 \%$ \\
\hline & Major & 30 & $29.3 \%$ \\
\hline \multicolumn{2}{|c|}{ Procedure Related Complications } & $\mathrm{N}=116$ & $\%$ \\
\hline Wound Infection & Yes & 34 & $29.3 \%$ \\
\hline $\begin{array}{c}\text { Intra-Abdominal Collec- } \\
\text { tion }\end{array}$ & Yes & 5 & $4.3 \%$ \\
\hline Peritonitis /Sepsis & Yes & 9 & $7.8 \%$ \\
\hline Peristomal Leak & Yes & 20 & $17.2 \%$ \\
\hline Tube Replacement & Yes & 11 & $9.5 \%$ \\
\hline Tube Block/Dislodge & Yes & 15 & $12.9 \%$ \\
\hline GI Symptoms & Yes & 25 & $21.6 \%$ \\
\hline
\end{tabular}

Table 1: Clinical burden associated with feeding enterostomies.

Subgroup analysis between feeding gastrostomy and feeding jejunostomy (Summarized in table 2) showed morbidities (40.9\% vs. $69 \%$. OR 0.311, P-Value 0.002) prolong hospital stay ( $5 \%$ vs. 16.3\%. OR 3.78, P-Value 0.042), ER visits (9.8\% vs. 23.6\%. OR 0.35, P-Value 0.039$)$ and OPD visits (11.4\% vs. $32.7 \%$ OR .26, P-Value 0.05 ) statistically significant.

\begin{tabular}{|c|c|c|c|c|c|}
\hline \multicolumn{2}{|c|}{ Parameters } & \multirow{2}{*}{$\begin{array}{c}\text { Gastros- } \\
\text { tomy } \\
n=61 \\
(52.5 \%) \\
37(60.6)\end{array}$} & $\begin{array}{c}\text { Jejunos- } \\
\text { tomy } \\
n=55 \\
(47.4 \%)\end{array}$ & \multirow{2}{*}{\begin{tabular}{|c|}
$\begin{array}{c}\text { P- } \\
\text { Value }\end{array}$ \\
0.033 \\
\end{tabular}} & \multirow{2}{*}{$\begin{array}{c}\text { Odd Ratio } \\
\text { 95\% CI } \\
2.14(1.02\end{array}$} \\
\hline Gender & Male & & 23 (41.8) & & \\
\hline & Female & $24(39.3)$ & 32 (58.1) & & $4.50)$ \\
\hline ER visit & Yes & $6(9.8)$ & 13 (23.6) & 0.039 & $\begin{array}{c}0.35(0.12- \\
1.00)\end{array}$ \\
\hline OPD visit & Yes & 7 (11.4) & 18 (32.7) & 0.05 & $\begin{array}{c}0.26(0.10- \\
0.70)\end{array}$ \\
\hline
\end{tabular}

\begin{tabular}{|c|c|c|c|c|c|}
\hline $\begin{array}{l}\text { Readmis- } \\
\text { sion }\end{array}$ & Yes & $4(6.50$ & $8(14.5)$ & 0.135 & $\begin{array}{c}0.41(0.11- \\
1.45)\end{array}$ \\
\hline \multirow{2}{*}{$\begin{array}{l}\text { Hospital } \\
\text { stay }\end{array}$} & $<4$ Days & $58(95.0)$ & $46(83.6)$ & \multirow[t]{2}{*}{0.042} & $3.78(0.96-$ \\
\hline & $>4$ Days & $3(5)$ & $9(16.3)$ & & 14.77) \\
\hline Morbidity & Yes & $25(40.9)$ & $38(69.0)$ & 0.002 & $\begin{array}{c}0.311(0.14- \\
0.66)\end{array}$ \\
\hline Mortality & Yes & $1(1.6)$ & $2(3.6)$ & 0.46 & $\begin{array}{c}0.44(0.039- \\
5.01)\end{array}$ \\
\hline $\begin{array}{c}\text { Complica- } \\
\text { tion }\end{array}$ & Yes & $25(4$ & 38 & 0.002 & ).14- \\
\hline $\begin{array}{l}\text { Wound } \\
\text { infection }\end{array}$ & Yes & $15(24.5)$ & 19 & 0.166 & $\begin{array}{c}0.61(0.27- \\
1.38)\end{array}$ \\
\hline $\begin{array}{c}\text { Peristomal } \\
\text { leak }\end{array}$ & Yes & $8(13.1)$ & .8) & 0.16 & $\begin{array}{c}0.54(0.21- \\
1.44)\end{array}$ \\
\hline Tube block & Yes & $2(3.2)$ & 3.6) & 0.001 & $\begin{array}{c}0.11(0.02- \\
0.51)\end{array}$ \\
\hline $\begin{array}{c}\text { Tube re- } \\
\text { placement }\end{array}$ & Yes & .5) & 7( & 0.20 & $\begin{array}{c}0.48(0.13- \\
0.511)\end{array}$ \\
\hline $\begin{array}{c}\text { Intra- } \\
\text { abdominal } \\
\text { collection }\end{array}$ & Yes & $3(4.9)$ & $2(3.6)$ & 0.54 & $\begin{array}{c}1.37(0.22- \\
8.52)\end{array}$ \\
\hline $\begin{array}{c}\text { Peritonitis/ } \\
\text { Sepsis } \\
\end{array}$ & Yes & $4(6.5)$ & $5(9.0)$ & 0.43 & $\begin{array}{c}0.70(0.17- \\
2.75)\end{array}$ \\
\hline $\begin{array}{l}\text { GI Symp- } \\
\text { toms }\end{array}$ & Yes & $8(13.1)$ & $17(30.9)$ & 0.017 & $\begin{array}{c}0.33(0.13- \\
0.86)\end{array}$ \\
\hline $\begin{array}{c}\text { Reopera- } \\
\text { tion }\end{array}$ & Yes & $2(3.2)$ & $1(1.8)$ & 0.539 & $\begin{array}{c}1.83(0.16- \\
20.76)\end{array}$ \\
\hline
\end{tabular}

Table 2: Comparison feeding gastrostomy vs feeding jejunostomy.

\section{Discussion}

Cachexia associated malnutrition in cancer patients warrant nutritional support. Parenteral and enteral nutrition are the two available sources but because of line related issues and need for long term nutritional support enteral nutrition is preferred over parenteral nutrition in cancer patients. In our institute due to limited resources surgically placed enteral feeding tubes are preferred over endoscopically or radiologically placed feeding tubes.

In this study we focused on surgically placed enteral tube related complication and clinical burden on health care professionals. The study demonstrated that 63 patients (54.3\%) develop tube related complications which is consistent with the available data [10]. The most common complication in our study was wound infection in $29.3 \%$ patients and GI symptoms in $21.6 \%$ patients. This high complication rate can be justified keeping in view cancer 
related cachexia, underlying comorbidities and general clinical condition of these patients [11,12]. Enteral feeding tubes are helpful in these patients to maintain nutritional requirements especially where oral intake is compromised like head and neck and upper GI track cancers [13].

This study suggested that tube related complications are not life threatening as total mortality was $2.6 \%$ but $54.3 \%$ morbidities with ER and OPD visits of $16.4 \%$ and $21.6 \%$ respectively high lightens the fact that clinical burden and health care resources utilization is significant. This burden not only effects health care professional but also compromise patients' quality of life, delay specific cancer related treatment as well as psychologically effect patient attendants.

Sun Z., et al. and Minarich MJ., et al. reported in their respective studies that surgically placed feeding jejunostomies have more complications in term of prolong hospital stay and frequent follow up visits in ER and OPD as well as tube related issues. Our study also reported the similar results and supported that feeding gastrostomies are far more better then jejunostomies in term of postoperative complications $[14,15]$. On the basis of similar results from our study and international data we recommend where possible feeding gastrostomies should be favored over feeding jejunostomies for nutritional support however to validate this concept a prospective study with large number of patients should be considered.

Our study has several limitations as it is a single centered retrospective study with recall bias for data collection. Furthermore, there is no fixed protocol in our institute for surgical technique, tube management and follow up of these patients that may influence the development of postoperative complications. In addition, patients may visit nearby hospital if they develop any complication and in such cases these complications will not be recorded in our system.

Our recommendations are for a prospective study with large number of patients. Golden AB., et al. also concluded the similar results and recommendations that clinical burden can be improved with patient and attendants education regarding tube management, developing a standardized feeding tube protocol which include standardized surgical technique, tube management and a proper follow up design and for which an institutional level patient care program should be initiated. So that tube related morbidities can be addressed earlier and managed accordingly hence avoid- ing serious complications. This will help to evaluate benefit versus morbidity related with this procedure [16].

\section{Conclusion}

Understanding the clinical burden and complications related with surgically placed feeding tubes is crucial as it will help in earlier identification and management of these issues and thus minimize associated morbidity and mortality.

Because of low clinical burden and complication feeding gastrostomy should be preferred over feeding jejunostomy where possible.

\section{Acknowledgement}

Special thanks to DR Muhammad Arsalan Khan Consultant HBP surgeon Sindh Institute of Urology and Transplantation for his valuable input regarding motivation and support for writing this article.

\section{Conflict of Interest}

Nothing to disclose.

\section{Bibliography}

1. Dann GC., et al. "An assessment of feeding jejunostomy tube placement at the time of resection for gastric adenocarcinoma: A seven-institution analysis of 837 patients from the U.S. gastric cancer collaborative". Journal of Surgical Oncology 112.2 (2015): 195-202.

2. Alvarez-Sarrado E., et al. "Feeding Jejunostomy after esophagectomy cannot be routinely recommended. Analysis of nutritional benefits and catheter-related complications". The American Journal of Surgery 217.1 (2019): 114-120.

3. Aoyagi T., et al. "Cancer cachexia, mechanism and treatment". World Journal of Gastrointestinal Oncology 7.4 (2015): 17-29.

4. Arends J., et al. "ESPEN guidelines on nutrition in cancer patients". Clinical Nutrition 36.1 (2017): 11-48.

5. Gyan E., et al. "Malnutrition in Patients With Cancer: Comparison of Perceptions by Patients, Relatives, and Physicians Results of the NutriCancer2012 Study". JPEN Journal of Parenteral and Enteral Nutrition 42.1 (2018): 255-260.

6. Mason MC., et al. "Preoperative cancer cachexia and shortterm outcomes following surgery". Journal of Surgical Research 205.2 (2016): 398-406. 
7. Patel SH., et al. "An assessment of feeding jejunostomy tube placement at the time of resection for gastric adenocarcinoma”. Journal of Surgical Oncology 107 (2013): 728-734.

8. Goldin AB., et al. "Emergency department visits and readmissions among children after gastrostomy tube placement". The Journal of Pediatrics 174 (2016): 1-9.

9. American Society for Parenteral and Enteral Nutrition (A.S.P.E.N.) Board of Directors. "Guidelines for the use of parenteral and enteral nutrition in adult and pediatric patients". JPEN Journal of Parenteral and Enteral Nutrition 33 (2002): 255-259.

10. Alvarez-Sarrado E., et al. "Feeding Jejunostomy after esophagectomy cannot be routinely recommended. Analysis of nutritional benefits and catheter-related complications". The American Journal of Surgery 2170.1 (2019): 114-120.

11. Arends J., et al. "ESPEN guidelines on nutrition in cancer patients". Clinical Nutrition 36.1 (2017): 11-48.

12. Gyan E., et al. "Malnutrition in Patients With Cancer: Comparison of Perceptions by Patients, Relatives, and Physicians Results of the NutriCancer2012 Study". Journal of Parenteral and Enteral Nutrition 42.1 (2018): 255-260.

13. Aoyagi T., et al. "Cancer cachexia, mechanism and treatment". World Journal of Gastrointestinal Oncology 7.4 (2015): 17-29.

14. Sun Z., et al. "Feeding jejunostomy tube placement during resection of gastric cancer". The Journal of Surgical Oncology Research 200 (2016): 189-194.

15. Minarich MJ and Schwarz RE. "Experience with a simplified feeding jejunostomy technique for enteral nutrition following major visceral operation". Translational Gastroenterology and Hepatology 3 (2018): 44.

16. Goldin AB., et al. "Emergency department visits and readmissions among children after gastrostomy tube placement". The Journal of Pediatrics 174 (2016): 1-9.

Volume 4 Issue 8 August 2021

(C) All rights are reserved by Muhammad Shadab Khan., et al. 